\title{
AKT mediates actinomycin D-induced p53 expression
}

\author{
Chih-Shou Chen ${ }^{1}$, Dong-Ru Ho ${ }^{1}$, Fei-Yun Chen ${ }^{2}$, Chang-Rong Chen ${ }^{2}$, Yu-De Ke ${ }^{2}$, \\ Jyan-Gwo Joseph Su² \\ ${ }^{1}$ Division of Urology, Department of Surgery, Chang Gung Memorial Hospital, Chiayi, Taiwan, ROC \\ 2 Department of Biochemical Science and Technology, National Chiayi University, Chiayi 600, Taiwan, ROC \\ Correspondence to: Jyan-Gwo Joseph Su, email: jgjsu@mail.ncyu.edu.tw.
}

Keywords: Actinomycin D, AKT, p53

Received: August 28, $2013 \quad$ Accepted: January 11,2014 Published: January 11, 2014

This is an open-access article distributed under the terms of the Creative Commons Attribution License, which permits unrestricted use, distribution, and reproduction in any medium, provided the original author and source are credited.

\section{ABSTRACT:}

\begin{abstract}
At high cytotoxic concentrations, actinomycin D (ActD) blocks transcription, decreasing levels of MDM2 and thus causing p53 stabilization. At low cytostatic concentrations, ActD causes ribosomal stress, which decreases MDM2 activity, resulting in p53 stabilization and activation. ActD can thus be used for p53-based cyclotherapy. We analyzed pathways mediating ActD-induced p53 expression. Inhibitors (LY294002, wortmannin, and deguelin) of phosphatidylinositol 3-kinases (PI3K) and AKT, but not inhibitors of MEK1/2, JNK, and p38-MAPK abolished the ActD-induced p53 expression in diverse cell types. RNA interference further supported these results. When AKT was downregulated by small hairpin RNA-AKTs, ActD-induced p53 expression was significantly decreased. ActD caused AKT phosphorylation at Ser473, indicating full activation of AKT. The potential for cancer therapy is discussed.
\end{abstract}

\section{INTRODUCTION}

In response to cellular stress such as DNA damage, oncogene activation, transcriptional inhibition, and hypoxia, tumor suppressor p53 is activated and expressed, and acts as a transcription factor to induce its target genes [1], thereby playing a central role in the regulation of DNA repair, cell cycle, apoptosis, senescence, and angiogenesis [2-4]. Its major target genes include proapoptotic genes Bax, Puma and Noxa, cell cycle regulator p21, and the senescence-inducing gene Plasminogen activator inhibitor 1 [5]. The restriction of cellular growth by $\mathrm{p} 53$ has been reported to result in cell cycle arrest or apoptosis [6], and targeting p53 and restoring p53 function to limit tumor growth has been intensively researched for cancer therapy [7]. AKT is a well-known survival factor that phosphorylates and activates oncoprotein HDM2 (also known as murine double minute 2 (MDM2), HDM2 in humans), and in turn, HDM2 induces degradation of p53 $[8,9]$. Thus AKT indirectly downregulates p 53 , and p53 negatively regulates AKT [10].

Actinomycin D (ActD), an antineoplastic antibiotic isolated from Streptomyces sp., has been reported to induce cytotoxicity and apoptosis, and inhibit growth of pancreatic cancer cells [11]. ActD inhibits cell proliferation by forming a stable complex with DNA duplexes via deoxyguanosine residues, resulting in the inhibition of RNA synthesis by blocking the elongation of RNA chains [12].

The application of ActD at high doses (> $800 \mathrm{nM})$ is limited due to its high toxicity through acting as a transcription blocker; however at low doses $(10-100 \mathrm{nM})$ it induces p53 expression and is not highly toxic $[13,14]$. In addition, at a high cytotoxic concentration $(50 \mathrm{nM})$, ActD has been shown to block transcription and decrease levels of HDM2 thereby stabilizing p53 [15]. Further, at a low cytostatic concentration $(2 \mathrm{nM})$, ActD causes ribosomal stress leading to a decrease in HDM2 activity and thereby p53 stabilization and activation. Therefore, combined treatment of low-dose ActD with other chemotherapeutic drugs may be a promising cancer therapy. The combined treatment of ActD with leptomycin B, a small molecule nuclear export inhibitor, has been shown to successfully lead to the accumulation of transcriptionally active p 53 in the nuclei of human papillomavirus positive cervical carcinoma cells, resulting in apoptosis of the cells [16]. Due to the inhibition of RNA transcription, ActD has been found to have antineoplastic properties in the treatment of various malignant neoplasms including Wilm's tumour [14]. In addition, ActD has been shown to mimic nutlin-3 in the activation of p53-dependent transcription, induction of a reversible protective growth arrest in normal cells, 
and enhancement of the activity of the chemotherapeutic drugs, melphalan and etoposide, resulting in apoptosis of p53 positive human tumor cells [14].

Although low doses of ActD have been studied in p53 base cyclotherapy, the kinase pathway by which ActD induces p53 has not been examined. Cyclotherapy may be achieved by combining ActD treatment with other drugs $[17,18]$, and therefore understanding the cellular kinase pathway for the drugs used in combination treatment will be valuable for future cyclotherapy studies. The present study analyzed the kinase pathway through which ActD induces p53, and found that AKT was phosphorylated and activated by ActD. AKT is required in mediating ActDinduced p53 expression. Thus, there is a novel function of ActD in the upregulation of AKT-mediated p53 expression. This study clarifies the signaling pathway that induces $\mathrm{p} 53$ via ActD, a potential chemotherapeutic agent.

\section{RESULTS}

\section{ActD dose- and time course-dependently induces protein expression and phosphorylation of p53}

Treatment with ActD (10 nM) distinctly induced the expression and phosphorylation of $\mathrm{p} 53$ at $18 \mathrm{~h}$, reaching a maximal response at $24 \mathrm{~h}$, and maintaining a high level of p53 for up to $30 \mathrm{~h}$ in the 293 and $293 \mathrm{~T}$ cells (Fig. 1A). In contrast, treatment with ActD $(10 \mathrm{nM})$ distinctly induced the expression and phosphorylation of p53 at $3 \mathrm{~h}$, reaching a maximal response at $6 \mathrm{~h}$, and maintaining a high level of p53 for up to $12 \mathrm{~h}$ in the HepG2 cells. In the Hepa-1c1c7 cells, treatment with ActD $(10 \mathrm{nM})$ distinctly induced the expression and phosphorylation of p53 at $6 \mathrm{~h}$, and a high level of p53 was maintained for up to $12 \mathrm{~h}$ (Fig. 1A). In the dosage studies, the expression and phosphorylation of p53 reached a maximal level with treatment of $10 \mathrm{nM}$ ActD for $24 \mathrm{~h}$ in the 293 and 293T cells (Fig. 1B). In the HepG 2 cells, the expression of p53 reached a maximal level with treatment of $10 \mathrm{nM}$ ActD, and decreased with doses of $100 \mathrm{nM}$ or higher for $6 \mathrm{~h}$ (Fig. 1B). Although p53 protein levels decreased after reaching the maximal level with treatment of $30 \mathrm{nM}$ ActD, phosphorylation of p53 still increased with treatment of high doses (100 and $300 \mathrm{nM}$ ) of ActD. In the Hepa1c1c7 cells, the expression of p53 reached a maximal level with treatment of $100 \mathrm{nM}$ ActD, and decreased with a dose of $300 \mathrm{nM}$ for $6 \mathrm{~h}$ (Fig. 1B). Although p53 protein levels decreased after reaching the maximal level with treatment of $100 \mathrm{nM}$ ActD, phosphorylation of p53 still increased with treatment of a high dose (300 nM) of ActD.

When the expression and phosphorylation patterns of HDM2 were examined, the results showed that HDM2 expression was induced by low doses of ActD ( 5 or 10 $\mathrm{nM})$, and suppressed by high doses ( $>10 \mathrm{nM})$ of ActD

A
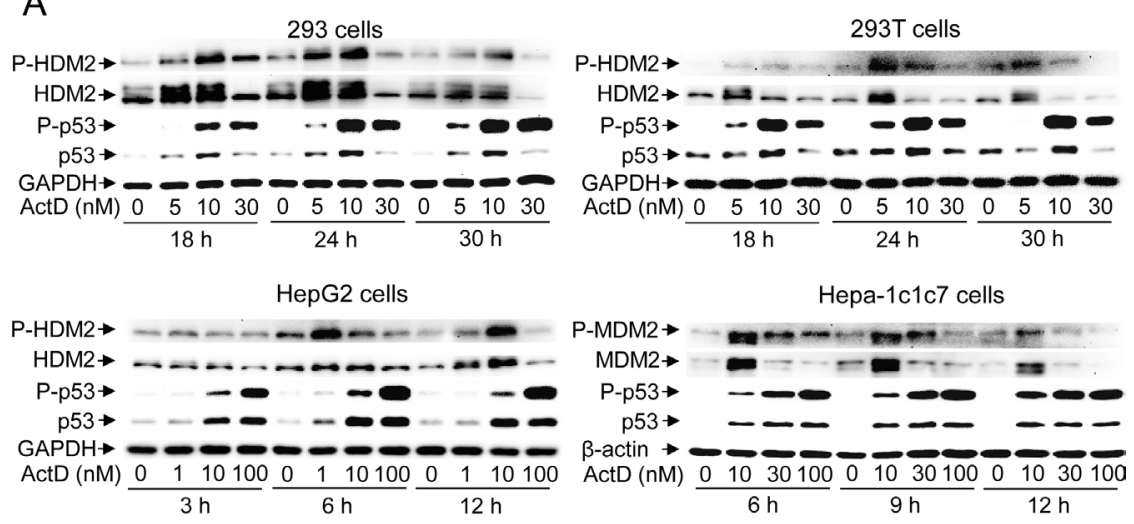

B
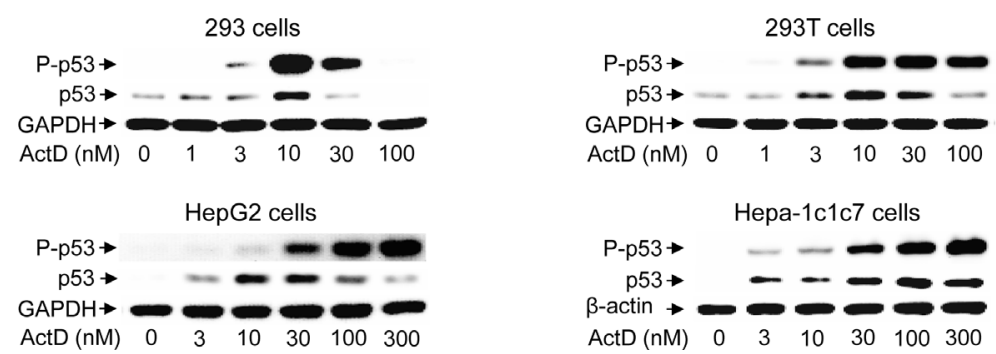

Figure 1: Time course and dose effect of the induction of p53 expression by actinomycin D (ActD). (A) 293 and $293 \mathrm{~T}$ cells were treated with $\operatorname{ActD}(0,5,10$ and $30 \mathrm{nM})$ for 18, 24, and $30 \mathrm{~h}$. HepG2 cells were treated with ActD $(0,1,10$ and $100 \mathrm{nM})$ for 3, 6, and $12 \mathrm{~h}$. Hepa-1c1c7 cells were treated with $\operatorname{ActD}(0,10,30$ and $100 \mathrm{nM})$ for 6, 9, and $12 \mathrm{~h}$. (B) 293 and 293T cells were treated with ActD $(0,1,3,10,30$ and $100 \mathrm{nM})$ for $24 \mathrm{~h}$. HepG2 and Hepa-1c1c7 cells were treated with ActD $(0,3,10,30,100$ and $300 \mathrm{nM})$ for $6 \mathrm{~h}$. The cells were then harvested, and cell lysates were analyzed by Western blotting using antibodies against p53, phospho-p53 (Ser15), HDM2, phosphor-HDM2 (Ser166), GAPDH, and $\beta$-actin. 
(Fig. 1A). In contrast, p53 expression was still induced at higher doses of ActD (>10 nM). The phosphorylation patterns of HDM2 were similar to the protein expression patterns of HDM2.

\section{ActD stimulates p53 activity}

A reporter plasmid, p53-TA-Luc, was used to quantify p53 activity. The p53 activity in the 293 and 293 T cells increased from 12 through $24 \mathrm{~h}$ after treatment with $10 \mathrm{nM}$ ActD (Fig. 2). The increase in the p53 activity in the HepG2 cells was also time course-dependent and reached a plateau at $12 \mathrm{~h}$ after treatment with $10 \mathrm{nM}$ ActD.
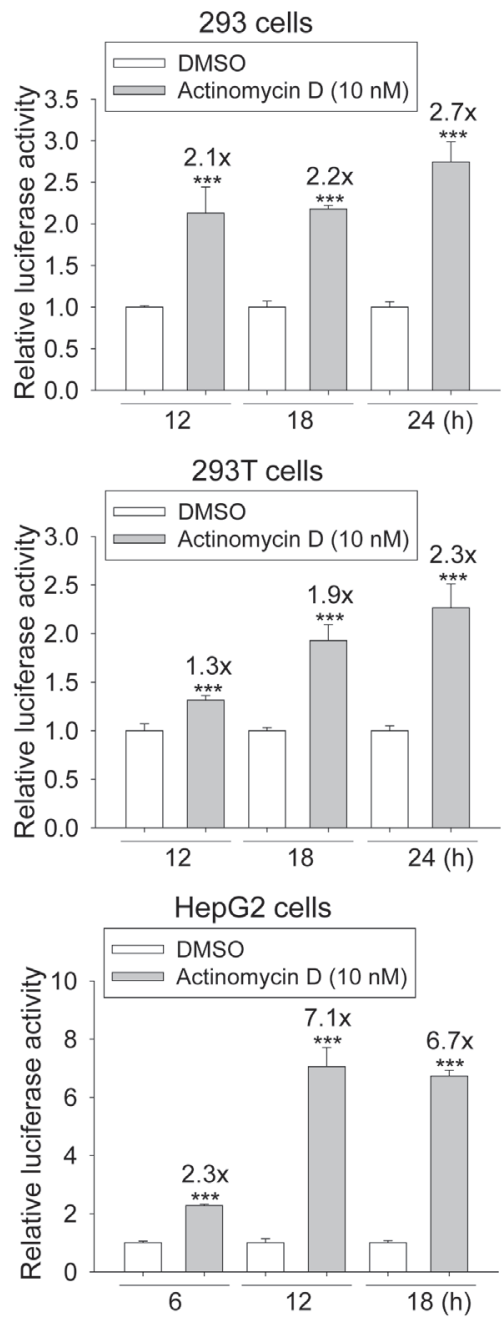

Figure 2: Induction of the transcriptional activity of p53 response element (p53RE) by actinomycin D (ActD) treatment. 293, 293T, and HepG2 cells were transfected with plasmids of p53-TA-Luc plus RSV-lacZ, and then incubated with $\mathrm{ActD}(10 \mathrm{nM})$ for the indicated time periods, followed by the activity assays of luciferase and $\beta$-galactosidase. Each experiment was assayed in triplicate and repeated at least three times. ${ }^{* * *}$ Indicates $p<0.001$ compared with the negative controls.

\section{The PI3K-AKT pathway mediates ActD-induced p53 expression}

We were interested in analyzing whether any kinase pathway mediated ActD-induced p53 expression and phosphorylation. Phosphatidylinositol-3-kinase (PI3K) inhibitors (LY294002 $(10 \mu \mathrm{M})$ and wortmannin $(10 \mu \mathrm{M}))$
A

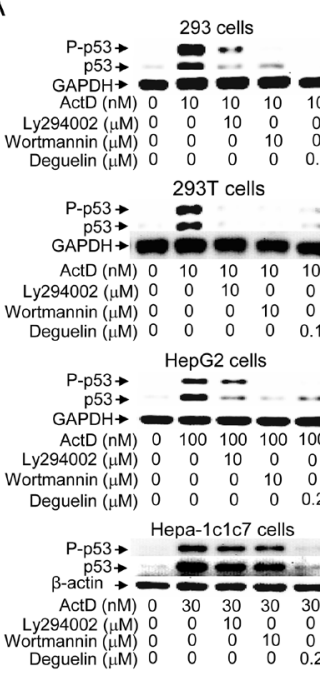

C

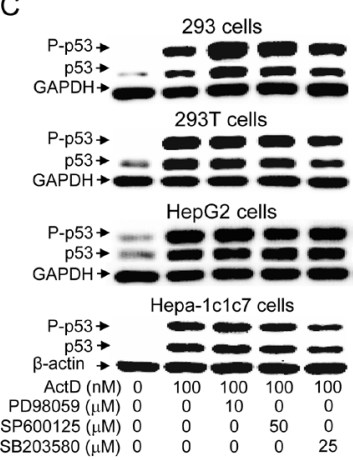

B

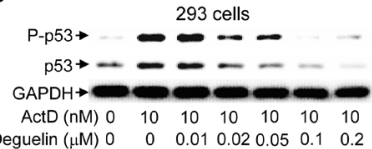

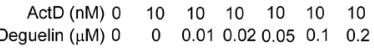

293T cells

$\rightarrow \infty-\infty$

$\begin{array}{llllllll}\text { ActD (nM) } & 0 & 10 & 10 & 10 & 10 & 10 & 10\end{array}$ Deguelin $(\mu \mathrm{M}) \quad 0 \quad 0 \quad 0.010 .020 .05 \quad 0.1 \quad 0.2$

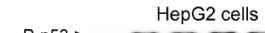

P-p53 $\rightarrow \infty-\infty-\cdots$

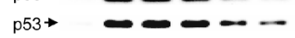

GAPDH $\rightarrow-2-$

ActD (nM) $0 \quad 100100100100100100$ Deguelin $(\mu \mathrm{M}) 0 \quad 0 \quad 0.010 .020 .05 \quad 0.1 \quad 0.2$
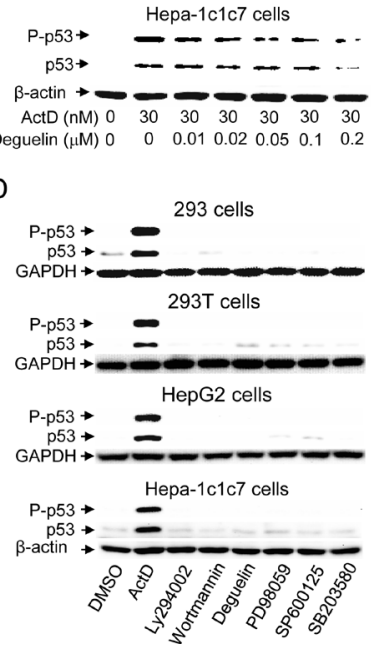

$\underset{\mathrm{P53} \rightarrow}{\longrightarrow}--<-$

Figure 3: Effects of kinase inhibitors on actinomycin D (ActD)-induced p53 expression. (A) Cells were pretreated with PI3K inhibitors (LY294002 $(10 \mu \mathrm{M})$ or wortmannin $(10$ $\mu \mathrm{M})$ ), and an AKT inhibitor (deguelin) for $1 \mathrm{~h}$, followed by treatment with ActD for $24 \mathrm{~h}$ in 293 and 293T cells, and for $6 \mathrm{~h}$ in HepG2 and Hepa-1c1c7 cells. (B) Cells were pretreated with deguelin, $(0.01,0.02,0.05,0.1$, and $0.2 \mu \mathrm{M})$ for $1 \mathrm{~h}$, followed by treatment with ActD for $24 \mathrm{~h}$ in 293 and 293T cells, and for $6 \mathrm{~h}$ in HepG2 and Hepa-1c1c7 cells. (C) The MEK1/2 inhibitor (PD98059, $10 \mu \mathrm{M}$ ), JNK inhibitor (SP600125, $50 \mu \mathrm{M}$ ), and p38 inhibitor (SB203580, $25 \mu \mathrm{M}$ ) were applied. Cells were pretreated with kinase inhibitors for $1 \mathrm{~h}$, followed by treatment with ActD for $24 \mathrm{~h}$ in 293 and 293T cells, and for $6 \mathrm{~h}$ in HepG2 and Hepa1c1c7 cells. (D) Cells were treated individually with ActD (10 $\mathrm{nM})$, LY294002 $(10 \mu \mathrm{M})$, wortmannin $(10 \mu \mathrm{M})$, deguelin $(0.2$ $\mu \mathrm{M}$ for 293, HepG2, and Hepa-1c1c7 cells, and $0.1 \mu \mathrm{M}$ for 293 T cells), PD98059 $(10 \mu \mathrm{M})$, SP600125 $(50 \mu \mathrm{M})$, and SB203580 $(25 \mu \mathrm{M})$ for $24 \mathrm{~h}$ in 293 and $293 \mathrm{~T}$ cells, and for $6 \mathrm{~h}$ in HepG2 and Hepa-1c1c7 cells. The cells were then harvested and cell lysates were analyzed by Western blotting using antibodies against $\mathrm{p} 53$, phospho-p53 (Ser15), GAPDH, and $\beta$-actin. 
and an AKT inhibitor (deguelin $(0.1$ or $0.2 \mu \mathrm{M})$ ) were added to the 293, 293T, HepG2, and Hepa-1c1c7 cells that had been treated with ActD. All of these inhibitors

A

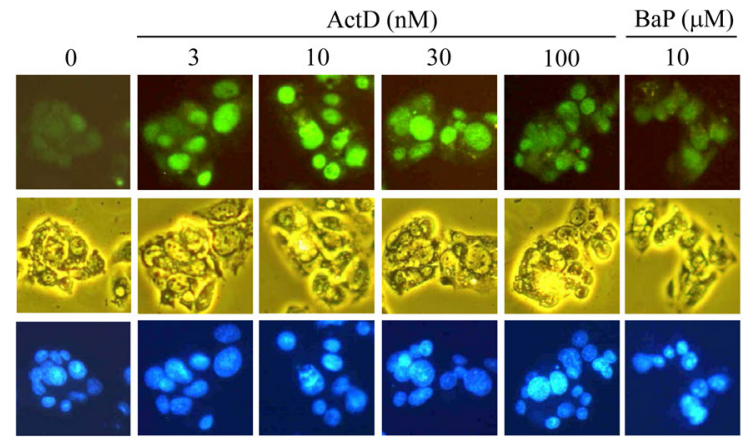

B

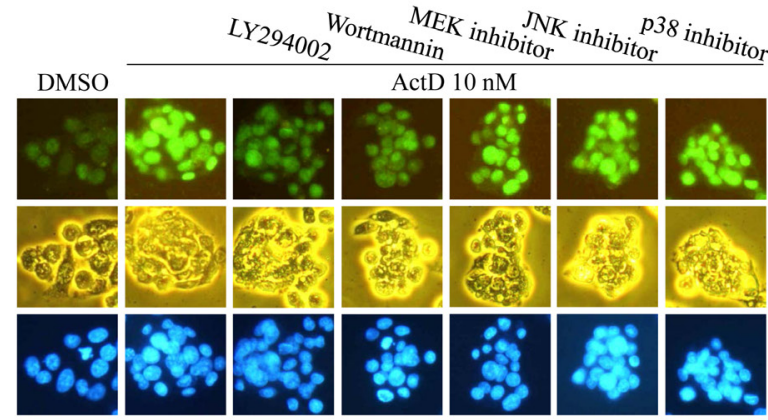

C $\quad \operatorname{ActD}(\mathrm{nM}) \quad 0$ Deguelin $(\mu \mathrm{M}) \quad 0$
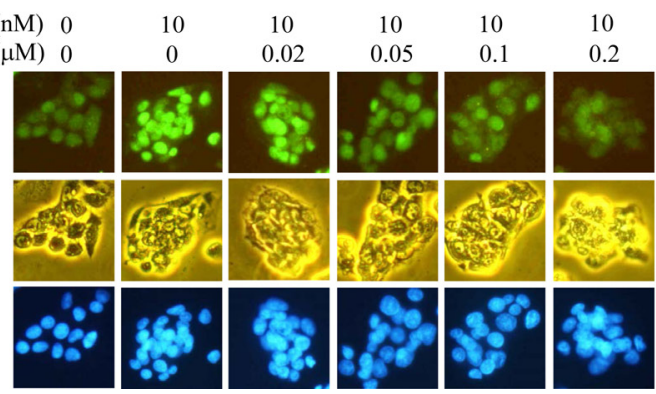

Figure 4: Actinomycin D(ActD)-induced p53 expression as revealed by immunofluorescence imaging. $\mathrm{HepG} 2$ cells were seeded on microscope cover glasses in 6-well plates overnight before drug treatment. (A) Cells were treated with $\operatorname{ActD}(3,10,30$, and $100 \mathrm{nM})$ and benzo[a]pyrene $(\mathrm{BaP})(10$ $\mu \mathrm{M})$ for $6 \mathrm{~h}$. (B) The PI3K inhibitors, (LY294002 $(10 \mu \mathrm{M})$ and wortmannin $(10 \mu \mathrm{M})$ ), MEK1/2 inhibitor (PD98059, $10 \mu \mathrm{M})$, JNK inhibitor (SP600125, $50 \mu \mathrm{M})$, and p38 MAPK inhibitor (SB203580, $25 \mu \mathrm{M}$ ) were applied. Cells were pretreated with kinase inhibitors for $1 \mathrm{~h}$, followed by treatment with ActD for 6 $\mathrm{h}$ in HepG2 cells. (C) Cells were pretreated with AKT inhibitor (deguelin, $0.02,0.05,0.1$, and $0.2 \mu \mathrm{M}$ ) for $1 \mathrm{~h}$, followed by treatment with ActD $(10 \mathrm{nM})$ for $6 \mathrm{~h}$. The cells were then fixed with ethanol. The expression of the 553 protein was probed using an antibody against $\mathrm{p} 53$, as revealed by fluorescence of goat polyclonal secondary antibody to mouse IgG-H\&L (DyLight ${ }^{\circledR}$ 488). The fluorescence emitted by the cells was viewed using a fluorescence microscope, equipped with U-MWB2 optical filters at excitation/emission wavelengths of $460 \sim 490 / 520 \mathrm{~nm}$. Nuclei were stained with Hoechst $33342(5 \mu \mathrm{g} / \mathrm{ml})$ and observed by a fluorescence microscope equipped with U-MWU optical filters with a U-MWU optical filter at an excitation wavelength of 355 $\mathrm{nm}$ and an emission wavelength of $420 \mathrm{~nm}$. The morphologies of the cells were examined by phase-contrast microscopy. either abolished or highly decreased the ActD-induced p53 expression (Fig. 3A). Deguelin dose-dependently suppressed p53 expression, and $0.05 \mu \mathrm{M}$ deguelin decreased the p53 expression in the 293, 293T and HepG2 cells (Fig. 3B). However, $0.2 \mu \mathrm{M}$ deguelin was required to cause a distinct decrease in p53 expression in the Hepa-1c1c7 cells. Inhibitors of mitogen-activated protein kinases (MAPKs), including MEK1/2 inhibitor (PD98059) and Jun N-terminal kinase inhibitor (SP600125), did not suppress the ActD-induced p53 expression or phosphorylation (ser15) (Fig. 3C). p38 inhibitor (SB203580) caused a minor reduction in the ActD-induced increase in the expression and phosphorylation of p53 in the 293T and Hepa-1c1c7 cells, but not in the 293 and HepG2 cells. The different results for the p38 inhibitor may be due to cell-specific and species-specific factors. None of the applied kinase inhibitors themselves distinctly induced p53 expression (Fig. 3D).

In order to further confirm the results of Western blotting, the expression of p53 was further examined by
DMSO

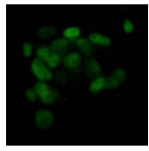

ActD
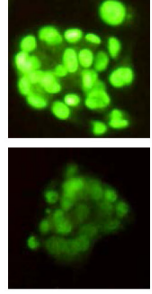

Wortmannin

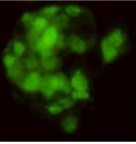

Deguelin
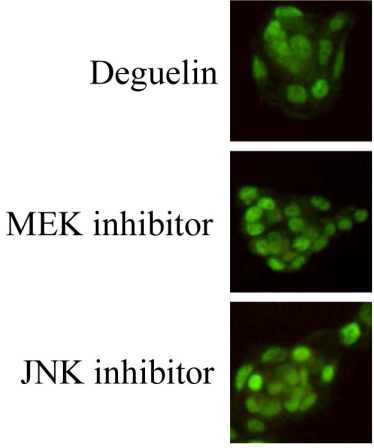

p38 inhibitor

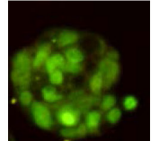

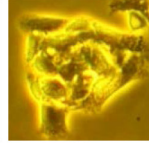
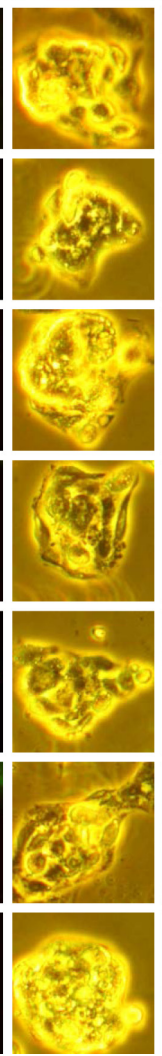
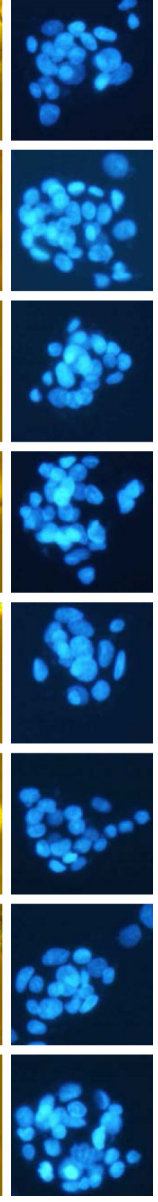

Figure 5: Effects of kinase inhibitors on $\mathrm{p} 53$ expression as revealed by immunofluorescence imaging. HepG2 cells were seeded on microscope cover glasses in 6-well plates overnight before drug treatment. Cells were treated individually with ActD $(10 \mathrm{nM})$, LY294002 $(10 \mu \mathrm{M})$, wortmannin $(10 \mu \mathrm{M})$, deguelin $(0.2 \mu \mathrm{M})$, PD98059 $(10 \mu \mathrm{M})$, SP600125 $(50 \mu \mathrm{M})$, and SB203580 $(25 \mu \mathrm{M})$ for $6 \mathrm{~h}$ in HepG2 cells. 
immunofluorescence staining. The HepG2 cells were treated with ActD for $6 \mathrm{~h}$, and the p53 expression level was examined by immunofluorescence imaging. The benzo $[a]$ pyrene $(\mathrm{BaP})$-induced $\mathrm{p} 53$ expression was used as a positive control, and the blue fluorescence dye, Hoechst 33342, revealed the location of the nuclei. Fluorescence was dose-dependently increased by treatment with ActD (3-30 nM), but decreased by treatment with $100 \mathrm{nM}$ ActD (Fig. 4A). These results were similar to those derived from Western blotting (Fig. 1B). PI3K inhibitors (LY294002 (10 $\mu \mathrm{M})$ and wortmannin $(10 \mu \mathrm{M}))$, but not MEK1/2 inhibitor (PD98059), JNK inhibitor (SP600125), or p38 MAPK inhibitor (SB203580), inhibited the ActD-induced p53 expression in the immunofluorescence assays (Fig. 4B). A dose of $50 \mathrm{nM}$ and higher of deguelin suppressed the ActD-induced p53 expression in the immunofluorescence assays (Fig. 4C). These results were also similar to those derived from Western blotting (Fig. 3B and C). When the cells were treated with each kinase inhibitor individually, the applied kinase inhibitors themselves caused a minor induction of p53 expression (Fig. 5).

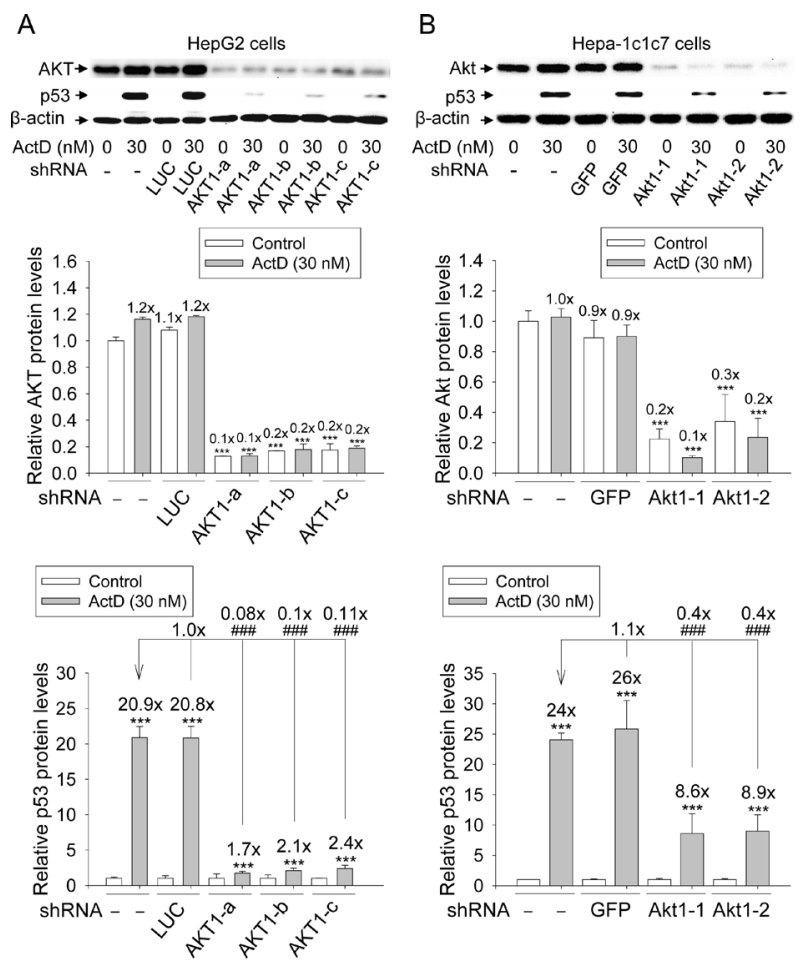

Figure 6: Effects of AKT RNAi on actinomycin D (ActD)-induced p53 expression. (A) HepG2 cells, with and without shRNA-LUC and shRNA-AKT1 were treated with ActD $(30 \mathrm{nM})$ for $6 \mathrm{~h}$. (B) Hepa-1c1c7 cells with and without shRNA-GFP and shRNA-Akt1 were treated with ActD $(30 \mathrm{nM})$ for $6 \mathrm{~h}$. The AKT and $\mathrm{p} 53$ protein levels revealed by Western blotting were quantified and standardized against the amount of $\beta$-actin protein. The results are expressed as the mean \pm SD $(\mathrm{n}=3) .{ }^{* *} p<0.001$ and $^{\# \# \# p<0.001 .}{ }^{*}$ A comparison with DMSOtreated cells without shRNA. ${ }^{*}$ A comparison with ActD-treated cells without shRNA.

\section{AKT is needed in the ActD induction of p53 expression}

To determine whether AKT was needed in the ActD induction of p53 expression, shRNAs for AKT1 were introduced into the HepG2 and Hepa-1c1c7 cells by lentiviruses to knock down AKT levels. Lentiviruses were used to individually produce the shRNAs of the green fluorescence protein (GFP), firefly luciferase (LUC), three different shRNAs of human AKT1 (AKT1-a, -b, and -c), and two different shRNAs of mouse Aktl (Akt1-1 and -2) in the virus-infected cells. Viruses with shRNA-LUC and shRNA-GFP were used as the controls of viral infection.

The relative AKT and p53 protein levels in the cells with and without shRNA-LUC, shRNA-GFP, and shRNA-AKT1, were demonstrated by Western blotting. There was no significant difference in AKT protein level between the cells without shRNA and those with shRNALUC or shRNA-GFP (Fig. 6A and B). Only 10-20\% and $10-30 \%$ of AKT was left in HepG2 and Hepa-1c1c7 cells with shRNA-AKT, respectively.

ActD $(30 \mathrm{nM})$ highly induced $\mathrm{p} 53$ protein in the cells without shRNA and in the cells with shRNA-LUC and shRNA-GFP (Fig. 6A and B). In contrast, the ActD-

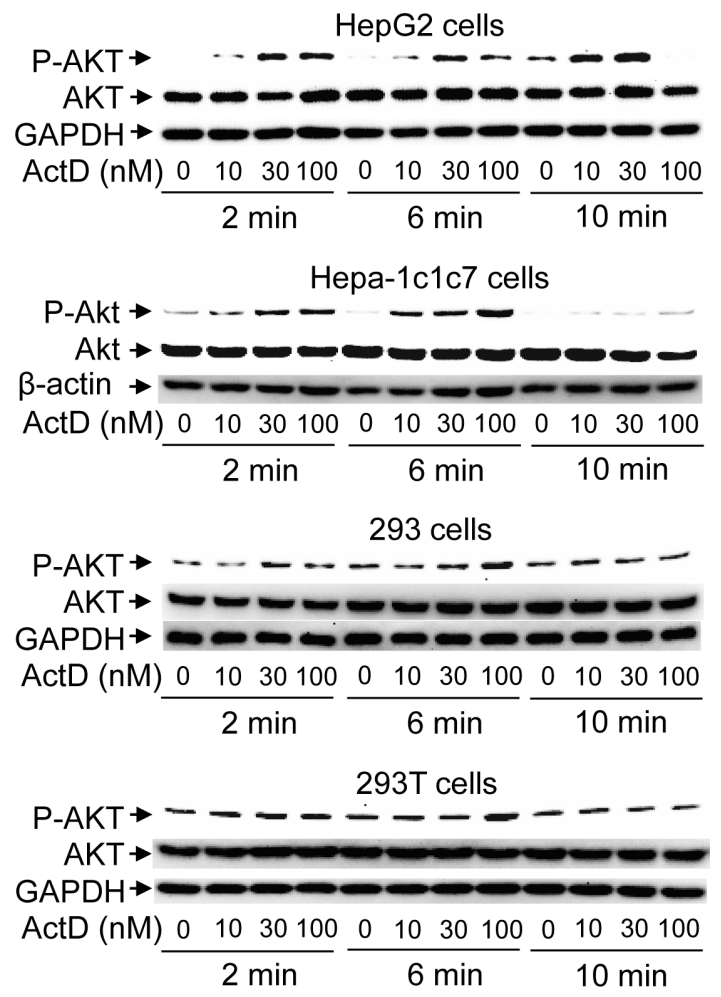

Figure 7: Phosphorylation of AKT induced by actinomycin D (ActD). (A) 293, 293T, HepG2, and Hepa$1 \mathrm{c} 1 \mathrm{c} 7$ cells were treated with $\operatorname{ActD}(0,10,30$ and $100 \mathrm{nM})$ for 2, 6, and 10 minutes ( $\mathrm{min})$. The cells were then harvested, and cell lysates were analyzed by Western blotting using antibodies against Akt and anti-phospho-Akt (Ser473), GAPDH, and $\beta$-actin. 
induced p53 protein levels in the HepG2 and Hepa-1c1c7 cells with shRNA-AKT were only $8-11 \%$ and $40 \%$, respectively, of that in the cells without shRNA (Fig. 6A and $\mathrm{B})$.

\section{ActD induces phosphorylation of AKT}

In order to further identify whether the ActDinduced p53 expression was mediated by the AKT pathway, phosphorylation at serine 473 of AKT was analyzed. Treatment with $10 \mathrm{nM}$ ActD for 2 and 6 minutes distinctly phosphorylated AKT at serine 473 in the HepG2 and Hepa-1c1c7 cells, however 30 and 100 $\mathrm{nM}$ ActD was required for distinct phosphorylation of AKT in the 293 and 293T cells, respectively (Fig. 7).

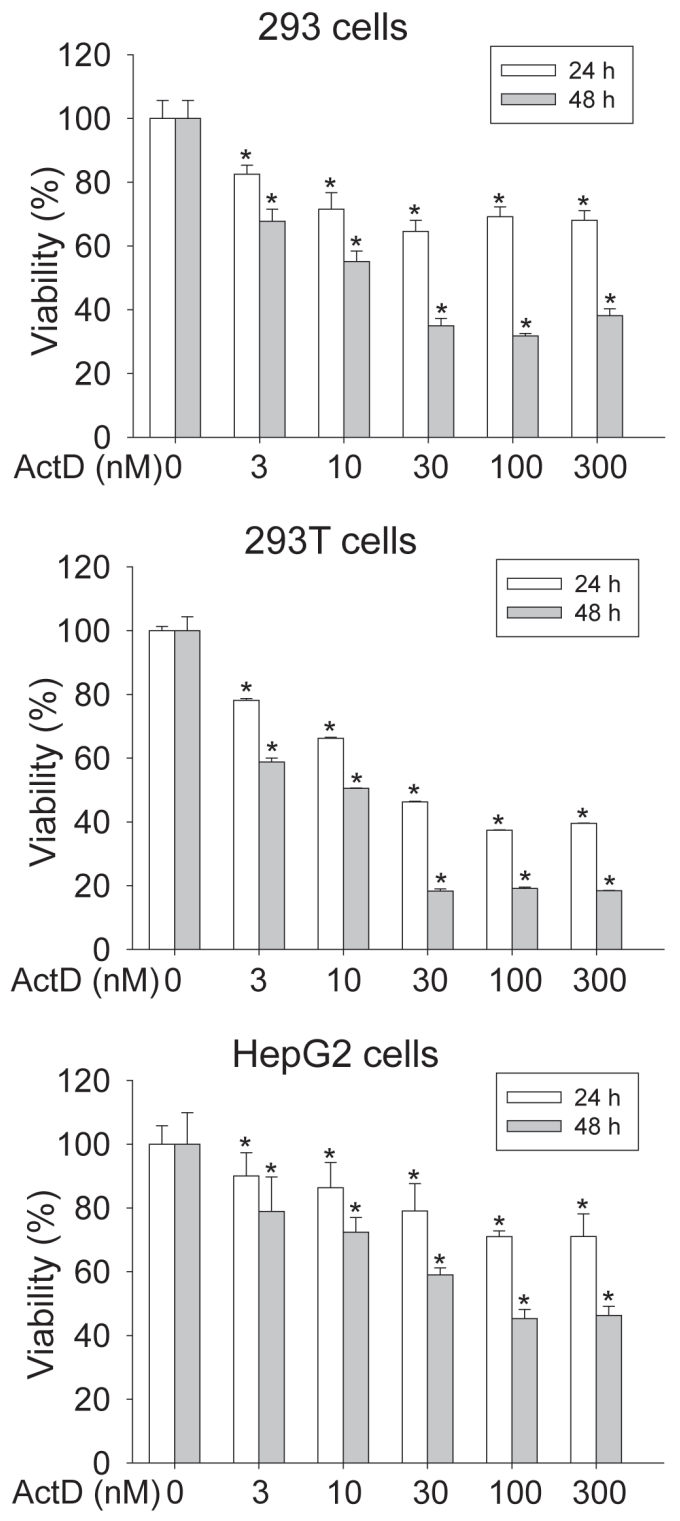

Figure 8: Influence on cell viability by actinomycin D (ActD). 293, 293T, and HepG2 cells were treated with ActD for 24 and $48 \mathrm{~h}$. Cell viability was analyzed by a MTT assay. Data are expressed as the mean $\pm \mathrm{SD}, n=6 .{ }^{*} p<0.05$.
Phosphorylation of AKT decreased after treatment with ActD for 10 minutes in the 293, 293T, and Hepa-1c1c7 cells, but still increased after treatment with ActD (10 and $30 \mathrm{nM}$ ) for 10 minutes in the HepG2 cells.

\section{ActD decreases cell viability}

A cell viability assay was then performed. Treatment with ActD at low doses of 3-30 nM for the 293 and 293 T cells, and 3-100 nM for the HepG2 cells, for 24 and 48 hours dose-dependently decreased cell viability (Fig. 8). However, treatment with ActD of 30-300 nM for the 293 and $293 \mathrm{~T}$ cells, and 100 and $300 \mathrm{nM}$ for the HepG2 cells, for 24 and 48 hours significantly decreased cell viability by a similar level.

\section{DISCUSSION}

A high dose of ActD has been reported to cause DNA damage and suppress transcription from all three classes of RNA polymerases, and ActD at a low dose has been reported to selectively block RNA poly I-dependent transcription [19]. However, ActD has been shown to induce p53 expression through ribosomal stress $[15,20]$. It has also been reported that the ribosomal protein L11 interacts with oncoprotein HDM2 and inhibits the function of HDM2, thus leading to the stabilization and activation of p53 [20-22]. In addition, the interaction of L11 with HDM2 has been shown to be enhanced in response to ActD $[19,21]$. ActD at a low dose has been reported to induce p53, and in turn, p53 increase the expression of HDM2 [15]. HDM2 has been shown to be transcribed by p53, which forms a negative feedback loop to promote poly-ubiquitination and degradation of p53 [23]. Although HDM2 is induced indirectly by ActD, ActD also induces the interaction of L11 with HDM2 leading to inhibition of HDM 2 function. Therefore p53 induced by ActD is more stable than that induced by other drugs without inhibiting HDM2 function. Accordingly, it is worth exploring the role of ActD as a p53 activator to reduce the growth of tumors.

Although it has been reported that ActD induces p53 expression, the kinase pathway for ActD-induced p53 has not been identified. Our results showed that inhibitors of PI3K and AKT, but not inhibitors of MEK1/2, JNK or p38, abolished ActD-induced p53 expression. MEK1/2 is immediately upstream of extracellular signal-regulated kinase (ERK) 1/2, and has been reported to activate ERK1/2 [24]. When a MEK1/2 inhibitor was applied, ERK1/2 activity was also inhibited. These kinase inhibitors of MEK1/2, JNK or p38 themselves did not distinctly induce $\mathrm{p} 53$ expression. The Western blotting results were further confirmed by in situ immunofluorescence staining. Phosphorylation of Ser15 in p53 is a sign that p53 is activated [25]. It has been shown that the MAP kinases 
including JNKs, ERKs, and p38 phosphorylate p53 in response to different stressful stimuli, and in turn, activate a p53 response leading to cell cycle arrest and apoptosis [24]. In addition, Ser15 of p53 has been shown to be phosphorylated by JNKs, ERKs, and p38 [24]. However, the ActD-induced phosphorylation (Ser15) of p53 was not inhibited by the inhibitors of p38, JNKs and ERKs in the current study, indicating that the phosphorylation (Ser15) of p53 was independent of these three MAP kinases.

Serine/threonine kinase AKT is a downstream kinase of PI3K and a critical PI3K effector [26]. AKT is recruited to plasma membranes by PtdIns(3,4,5)P3 (PIP3), and AKT is then phosphorylated on Thr-308 and Ser473 by PDK1 and mTORC2-Rictor, respectively. Upon Ser473 phosphorylation by mTORC2, AKT is fully activated [27]. Our results showed that ActD induced the phosphorylation of AKT at Ser473 within 2 minutes. In addition to PI3K inhibitors, deguelin, an AKT inhibitor, suppressed ActDinduced p53 expression in a dose-dependent manner. This confirms that ActD-induced p53 expression is mediated by the activation of AKT. Finally, the necessity of AKT in mediating ActD-induced p53 expression was confirmed by RNA interference on AKT. ActD-induced p53-expression significantly decreased when AKT expression was knocked-down by shRNA. Although AKT has been shown to downregulate p53 [28], the findings in the current study reveal a new function of AKT in the upregulation of $\mathrm{p} 53$.

PI3K-AKT signaling has been reported to promote the activation of the oncoprotein HDM2 and to downregulate the p53 tumor suppressor [28], and therefore AKT is defined as a survival factor. In contrast, AKT has been shown to be downregulated by p53 [10, 29]. However, our results showed that treatment with ActD immediately activated AKT signaling, and thereby induced p53 expression.

MAPKs have been widely reported to be involved in the activation of $\mathrm{p} 53$. The p38-mediated p53 phosphorylation at Ser15 and subsequent p53 induction has been reported to be responsible for apoptosis induced by nitric oxide, hypoxic conditions, DNA-damaging agents, 1-nitropyrene, and benzo[a]pyrene [30-32]. In response to oxidative stress, JNKs have been shown to phosphorylate p53 at Ser15 [33], and ERKs have been shown to mediate the p53 activation induced by colcemid and resveratrol $[34,35]$. AKT is a central point in cell signaling downstream of growth factors, cytokines, and other cellular stimuli, and thus it acts as a key regulator of a wide range of cellular processes including growth, proliferation and survival [9]. Therefore, AKT is best known for promoting cell survival and growth. In contrast to MAPKs, AKT inhibits the expression and function of apoptotic proteins, BAD and caspase-9, and promotes the activity of HDM2 which degrades p53 [9]. It has been reported that AKT and p53 counteract each other [3642]. In contrast, the results of the current study showed that AKT, but not MAPKs, mediated ActD-induced p53 expression and activation.

With regards to the induction of p53, the p53 protein expression peaked at $24 \mathrm{~h}, 24 \mathrm{~h}, 6 \mathrm{~h}$, and $6 \mathrm{~h}$ with $10 \mathrm{nM}, 10 \mathrm{nM}, 10$, and $100 \mathrm{nM}$ ActD treatment in the 293, 293T, HepG2, and Hepa-1c1c7 cells, respectively. Upon longer periods and higher doses of ActD the p53 expression decreased, which may be due to suppression of transcription from all three classes of RNA polymerases. Activated p53, a sequence-specific transcription factor, has been reported to bind to the p53RE on p53-targeted genes such as p21, to direct its downstream signals and actions [43]. In the current study, ActD also showed a time coursedependent increase in p53 activity, assayed by measuring the transcriptional activity of a p53RE reporter plasmid. Interestingly, ActD-induction of p53 expression was much faster in liver cells than in kidney cells.

HDM2 was transcribed by the p53 transcription factor, and the patterns of expression and phosphorylation of HDM2 were parallel to those of p53 at low doses (5 or $10 \mathrm{nM}$ ) of ActD treatment. However, p53, but not HDM2 expression was still induced by high doses $(>10 \mathrm{nM})$ of ActD treatment. Further studies are needed to elucidate the discrepancy between these expression patterns at high doses of ActD treatment.

ActD $(\leq 10 \mathrm{nM})$ dose-dependently decreased cell viability, and its induction of active p53 also peaked at $10 \mathrm{nM}$ in the 293 and $293 \mathrm{~T}$ cells. High doses ( $\geq 30 \mathrm{nM})$ of ActD did not further decrease cell viability or increase p53 expression. In contrast, ActD $(\leq 100 \mathrm{nM})$ dosedependently decreased cell viability and increased active p53 in the HepG2 cells. Therefore, the results of the cell viability assay reflected the activation of $\mathrm{p} 53$.

It has been reported that the activation of AKT involves ionizing radiation induction of p53 [44]. Constitutively expressed active myristoylated AKT has been reported to increase p53 levels [45]. However, ActD is the first chemical compound that has been shown to date to induce the AKT-mediated p53 expression.

An ideal chemotherapeutic agent is expected to specifically kill tumor cells, cause the least amount of undesirable side effect, and leave normal cells unharmed. However, the specificity of conventional chemotherapeutic drugs towards cancer cells is limited. Cyclotherapy is a prospective therapeutic strategy for the protection of normal cells from the side effects of chemotherapy [17]. The idea of p53-based cyclotherapy is that a p53 activator ceases proliferation in normal tissues while leaving the p53-deficient tumor susceptible to the toxicity of S- or M-phase chemotherapeutic poisons [18]. In addition, the p53 activator does not affect the sensitivity of tumor cells to the chemotherapeutic agents.

ActD has been shown to have a reversible cytostatic effect and the ability to arrest cells in both the G1 and G2 phase of the cell cycle [17]. A low, non-genotoxic, dose (about $1 \mathrm{nM}$ ) of ActD has been shown to induce a reversible cytostatic effect in normal proliferating dermal 
fibroblasts and protect them from the aneuploidy induced by the aurora kinase inhibitor VX-680 and the toxic effects of gemcitabine [46, 47]. In contrast, pretreatment of ActD did not weaken the growth inhibitory effects of VX-680 in clonogenic assays [46] and did not affect the sensitivity of the tumor cells to gemcitabine [47].

ActD is a classic clinically approved drug [46]. Based on studies on ActD, about 1-4 nM of ActD is suggested for cyclotherapy [18, 46, 47], and $3 \mathrm{nM}$ or higher may be appropriate for chemotherapy.

In summary, our findings showed that ActD induces the phosphorylation of AKT, thereby activating AKT. In turn, AKT signaling is essential in mediating ActD-induced p53 expression. The expression and phosphorylation (Ser15) of p53 is independent of JNKs, ERKs, and p38. Thus, upon activation by ActD, AKT becomes an inducer of p53 tumor suppression instead of being a survival factor, as consistently shown in human embryonic kidney cell lines (293 and 293T), human hepatoma cell line (HepG2), and mouse hepatoma cell line (Hepa-1c1c7). These findings on ActD signaling to induce p53 may be valuable for the development of treatment for human tumors.

\section{MATERIALS AND METHODS}

\section{Reagents and antibodies}

ActD, LY-294002, wortmannin, deguelin, PD98059, and Hoechst 33342 were obtained from Sigma (St. Louis, MO), and SB203580 and SP600125 were obtained from Selleckchem (Houston, TX). Minimum essential medium alpha (MEM $\alpha)$, MEM, Dulbecco's modified Eagle medium/nutrient mixture F12 (DMEM/F12), and fetal bovine serum (FBS) were obtained from Gibco (Grand Island, NY). Goat anti-mouse immunoglobulin $\mathrm{G}$ (IgG)-horseradish peroxidase (HRP), and goat antirabbit IgG-HRP were obtained from Santa Cruz Biotech (Santa Cruz, CA). Anti-glyceraldehyde 3 phosphate dehydrogenase (GAPDH), anti-p53, and goat polyclonal secondary antibodies to mouse IgG-H\&L (DyLight $\mathbb{R}$ 488) were obtained from Abcam (Cambridge, UK). Anti-phospho-p53 (ser15), antiphospho-HDM2 (ser166), anti-Akt and anti-phospho-Akt (Ser473) were obtained from Cell Signaling (Danvers, MA), and anti-HDM2 was obtained from Calbiochem (San Diego, CA). Serine/ threonine phosphatase inhibitor cocktail was obtained from Bionovas (Toronto, Ontario). T-Pro Non-liposomal Transfection Reagent II (NTRII) was obtained from JF JiFeng Biotechnology (Taipei, Taiwan). Fluoromount-G was obtained from Southern Biotech (Birmingham, Alabama).

\section{Cell cultures}

293 is a human embryonic kidney cell line, and $293 \mathrm{~T}$ is a derivative of 293 cells with an expression of SV40 T antigen. HepG2 is a human hepatoma cell line, and Hepa-1c1c7 is a mouse hepatoma cell line. The 293 and Hepa-1c1c7 cells were maintained in MEM $\alpha$, and the HepG2 cells in MEM plus 10\% heat-inactivated FBS, 2 $\mathrm{mM}$ L-glutamine, 100 units $/ \mathrm{ml}$ penicillin, and $100 \mu \mathrm{g} / \mathrm{ml}$ streptomycin. The 293 T cells were maintained in DMEM/ F12 plus 5\% heat-inactivated FBS, 2 mM L-glutamine, 100 units $/ \mathrm{ml}$ penicillin, and $100 \mu \mathrm{g} / \mathrm{ml}$ streptomycin. The cultured cells were kept at $37^{\circ} \mathrm{C}$ in a $95 \%$ air $/ 5 \%$ $\mathrm{CO}_{2}$ environment. The agents were dissolved in dimethyl sulfoxide (DMSO).

\section{Plasmid construction and reporter activity assay}

p53-TA-Luc contains a p53 response element (p53RE), located upstream of the minimal TA promoter (Clontech, Mountain View, CA). The other reporter (RSVlacZ) expresses a lacZ gene-encoded $\beta$-galactosidase, driven by a Rouse sarcoma virus long terminal repeat (LTR). Luciferase activity indicates transcriptional activity of the p53RE, and $\beta$-galactosidase activity of RSV-lacZ was used to normalize the luciferase activity. For DNA transfection, the HepG2, 293, and 293T cells were seeded respectively at $2 \times 10^{5}, 2 \times 10^{5}$, and $1 \times 10^{5}$ cells/well in 24-well plates overnight, and then the p53-TA-Luc luciferase reporter plasmid and RSV-lacZ plasmids were transiently transfected into the cells using NTRII for 6 $\mathrm{h}$, followed by treatment with the test compounds. Cell lysates were collected at the appropriate time points after treatment with the test compounds and were assayed for both luciferase and $\beta$-galactosidase activities using Britelite (PerkinElmer) and a Galacto-Star ${ }^{\mathrm{TM}}$ System (Tropix, Bedford, MA), respectively, according to the manufacturer's instructions.

\section{Western blotting}

The HepG2, Hepa-1c1c7, 293, and 293T cells were seeded respectively at $1 \times 10^{6}, 4 \times 10^{5}, 1.5 \times 10^{6}$, and $1.3 \times$ $10^{6}$ cells/6-cm dish overnight. The cells were then cultured with the test compounds for the appropriate time periods. At the end of the desired treatment times, cell lysates were prepared in lysis buffer (1\% NP-40, $0.5 \mathrm{mM}$ Tris$\mathrm{HCl}$ (pH 7.5), 0.14 M NaCl, $5 \mathrm{mM} \mathrm{KCl,} 5$ mM EDTA, and $1 \mathrm{mM}$ phenylmethylsulfonyl fluoride) plus serine/ threonine phosphatase inhibitor cocktail. Western blotting was performed as described previously [48]. 


\section{Immunofluorescence staining}

To detect the expression of p53, the HepG2 cells were seeded at $1 \times 10^{6}$ cells/well on microscope cover glasses in 6-well plates overnight before being treated with the drugs for the appropriate time periods, followed by washing with phosphate-buffered saline and fixing with ethanol. The expression of the p53 protein was probed using an antibody against p53, as revealed by fluorescence of a goat polyclonal secondary antibody to mouse IgG-H\&L (DyLight ${ }^{\circledR} 488$ ). The bisbenzimide dye, Hoechst $33342(5 \mu \mathrm{g} / \mathrm{ml})$, was then added to stain the chromosomes and reveal the location of the nuclei, and Fluoromount-G was added to reduce fluorochrome quenching during analysis of the slides by fluorescence microscopy. Fluorescence emitted by DyLight $\left({ }^{\circ} 488\right.$ was viewed using a fluorescence microscope (Olympus, Tokyo, Japan), equipped with U-MWB2 optical filters at excitation/emission wavelengths of 460-490/520 nm. The fluorescence emitted by Hoechst 33342 was viewed using a fluorescence microscope, equipped with U-MWU optical filters at excitation/emission wavelengths of 330-385/420 nm.

\section{RNA interference (RNAi)}

To perform RNAi and knock down AKT, 21 nucleotide duplexes corresponding to the human AKT1 mRNA (GenBank: NM_005163) and the mouse Akt1 mRNA (GenBank: NM_009652) were carried individually by lentiviruses (National RNAi Core Facility, Taipei, Taiwan). The three nucleotide duplexes for human AKT1 were AKT1-a (GCATCGCTTCTTTGCCGGTAT, clone ID: TRCN0000221554), AKT1-b (GATCCTC AAGAAGGAAGTCAT, clone ID: TRCN0000221553), and AKT1-c (CGCGTGACCATGAACGAGTTT, clone ID: TRCN0000221555). The two nucleotide duplexes for mouse Aktl were Akt1-1 (TCTGAGA CTGACACCAGGTAT, clone ID: TRCN0000022937) and Akt1-2 (GCACATCAAGATAACGGACTT, clone ID: TRCN0000022934).

Nucleotide duplexes for luciferase (LUC; CAAATCACAGAATCGTCGTAT, clone ID TRC N0000072246) and green fluorescence protein (GFP; TATCATGGCCGACAAGCA, clone ID: TRCN0000072180) were used as controls for viral infection. HepG2 cells $\left(1.6 \times 10^{5}\right.$ cells/well $)$ and Hepa$1 \mathrm{c} 1 \mathrm{c} 7\left(1 \times 10^{5} \mathrm{cells} /\right.$ well $)$ cells were seeded individually in 6 -well plates overnight and then infected by a lentivirus (4 $\times 10^{5} /$ well) for $24 \mathrm{~h}$. Forty-eight hours after infection, the HepG2 and Hepa-1c1c7 cells with shRNA were selected by 2 and $1 \mu \mathrm{g} / \mathrm{ml}$ puromycin, respectively, for $12 \mathrm{~h}$ to obtain stable infectants. The cells were then maintained in a medium containing puromycin $(0.5 \mu \mathrm{g} / \mathrm{ml})$.

\section{Determination of cell viability by MTT assay}

HepG2, 293, and 293T cells were seeded in 96-well plates at $4 \times 10^{3}, 1 \times 10^{4}$, and $1 \times 10^{4}$ cells, respectively, with $0.1 \mathrm{ml}$ medium. After overnight culture, they were treated with a range of concentrations of ActD for 24 and $48 \mathrm{~h}$, followed by incubation with methylthiazolyldiphenyl-tetrazolium bromide (MTT) (Sigma) for the assay. The optical density was detected at $550 \mathrm{~nm}$ using an enzyme-linked immunosorbent assay plate reader (BIO-TEK, Winooski, VT). Six samples were assayed for each experiment which was repeated at least three times.

\section{Statistical analysis}

Data are representative of at least three independent experiments under identical conditions and are expressed as the mean \pm standard deviation (SD). Differences in the data of the controls and further treatment with various compounds were analyzed using the Student's $t$-test. Statistical probability $(p)$ was expressed as $* p<0.05$, $* * * p<0.001$ and ${ }^{\# \#} p<0.001$. Means were considered significantly different at $p<0.05$.

\section{ACKNOWLEDGEMENTS}

This work was supported by a grant from Chang Gung Memorial Hospital, Chiayi Branch (CMRPG6A0051).

The authors declare no conflict of interest.

\section{REFERENCES}

1. Vousden $\mathrm{KH}$ and $\mathrm{Lu} \mathrm{X}$. Live or let die: the cell's response to p53. Nat Rev Cancer. 2002; 2(8):594-604.

2. Fridman JS and Lowe SW. Control of apoptosis by p53. Oncogene. 2003; 22(56):9030-9040.

3. Shangary S and Wang S. Targeting the MDM2-p53 interaction for cancer therapy. Clin Cancer Res. 2008; 14(17):5318-5324.

4. Teodoro JG, Evans SK and Green MR. Inhibition of tumor angiogenesis by p53: a new role for the guardian of the genome. J Mol Med. 2007; 85(11):1175-1186.

5. Kortlever RM, Higgins PJ and Bernards R. Plasminogen activator inhibitor-1 is a critical downstream target of p53 in the induction of replicative senescence. Nat Cell Biol. 2006; 8(8):877-884.

6. Haupt S, Berger M, Goldberg Z and Haupt Y. Apoptosis the p53 network. J Cell Sci. 2003; 116(Pt 20):4077-4085.

7. Wang W and El-Deiry WS. Restoration of p53 to limit tumor growth. Curr Opin Oncol. 2008; 20(1):90-96.

8. Downward J. PI 3-kinase, Akt and cell survival. Semin Cell 
Dev Biol. 2004; 15(2):177-182.

9. Manning $\mathrm{BD}$ and Cantley LC. AKT/PKB signaling: navigating downstream. Cell. 2007; 129(7):1261-1274.

10. Feng Z. p53 regulation of the IGF-1/AKT/mTOR pathways and the endosomal compartment. Cold Spring Harb Perspect Biol. 2010; 2(2):a001057.

11. Kleeff J, Kornmann M, Sawhney $\mathrm{H}$ and Korc $\mathrm{M}$. Actinomycin D induces apoptosis and inhibits growth of pancreatic cancer cells. Int J Cancer. 2000; 86(3):399-407.

12. Sobell HM. Actinomycin and DNA transcription. Proc Natl Acad Sci USA. 1985; 82(16):5328-5331.

13. Chen Y, Liu J, Yuan B, Cao C, Qin S, Cao X, Bian G, Wang $Z$ and Jiang J. Methylated actinomycin D, a novel actinomycin D analog induces apoptosis in HepG2 cells through fas- and mitochondria-mediated pathways. Mol Carcinog. 2013; 52(12):983-996.

14. Choong ML, Yang H, Lee MA and Lane DP. Specific activation of the $\mathrm{p} 53$ pathway by low dose actinomycin D: a new route to p53 based cyclotherapy. Cell Cycle. 2009; 8(17):2810-2818.

15. van Leeuwen IM, Higgins $M$, Campbell J, Brown CJ, McCarthy AR, Pirrie L, Westwood NJ and Lain S. Mechanism-specific signatures for small-molecule p53 activators. Cell Cycle. 2011; 10(10):1590-1598.

16. Hietanen S, Lain S, Krausz E, Blattner C and Lane DP. Activation of p53 in cervical carcinoma cells by small molecules. Proc Natl Acad Sci USA. 2000; 97(15):85018506.

17. Rao B, Lain S and Thompson AM. p53-Based cyclotherapy: exploiting the 'guardian of the genome' to protect normal cells from cytotoxic therapy. Br J Cancer. 2013; 109(12):2954-2958.

18. van Leeuwen IM. Cyclotherapy: opening a therapeutic window in cancer treatment. Oncotarget. 2012; 3(6):596600 .

19. Zhang Y, Wolf GW, Bhat K, Jin A, Allio T, Burkhart WA and Xiong Y. Ribosomal protein L11 negatively regulates oncoprotein MDM2 and mediates a p53-dependent ribosomal-stress checkpoint pathway. Mol Cell Biol. 2003; 23(23):8902-8912.

20. Miliani de Marval PL and Zhang Y. The RP-Mdm2-p53 pathway and tumorigenesis. Oncotarget. 2011; 2(3):234238.

21. Lohrum MA, Ludwig RL, Kubbutat MH, Hanlon M and Vousden KH. Regulation of HDM2 activity by the ribosomal protein L11. Cancer Cell. 2003; 3(6):577-587.

22. Holzel M, Burger K, Muhl B, Orban M, Kellner M and Eick D. The tumor suppressor p53 connects ribosome biogenesis to cell cycle control: a double-edged sword. Oncotarget. 2010; 1(1):43-47.

23. Toledo F and Wahl GM. Regulating the p53 pathway: in vitro hypotheses, in vivo veritas. Nat Rev Cancer. 2006; 6(12):909-923.

24. Wu GS. The functional interactions between the p53 and
MAPK signaling pathways. Cancer Biol Ther. 2004; 3(2):156-161.

25. Levine AJ. p53, the cellular gatekeeper for growth and division. Cell. 1997; 88(3):323-331.

26. Vasudevan KM and Garraway LA. AKT signaling in physiology and disease. Curr Top Microbiol Immunol. 2010; 347:105-133.

27. Liao Y and Hung MC. Physiological regulation of Akt activity and stability. Am J Transl Res. 2010; 2(1):19-42.

28. Mayo LD and Donner DB. The PTEN, Mdm2, p53 tumor suppressor-oncoprotein network. Trends Biochem Sci. 2002; 27(9):462-467.

29. Gottlieb TM, Leal JF, Seger R, Taya Y and Oren M. Crosstalk between Akt, p53 and Mdm2: possible implications for the regulation of apoptosis. Oncogene. 2002; 21(8):12991303.

30. Kim SJ, Hwang SG, Shin DY, Kang SS and Chun JS. p38 kinase regulates nitric oxide-induced apoptosis of articular chondrocytes by accumulating p53 via NFkappa B-dependent transcription and stabilization by serine 15 phosphorylation. J Biol Chem. 2002; 277(36):33501-33508.

31. Zhu Y, Mao XO, Sun Y, Xia Z and Greenberg DA. p38 Mitogen-activated protein kinase mediates hypoxic regulation of Mdm2 and p53 in neurons. J Biol Chem. 2002; 277(25):22909-22914.

32. Su JG, Liao PJ, Huang MC, Chu WC, Lin SC and Chang YJ. Aldo-keto reductase $1 \mathrm{C} 2$ is essential for 1-nitropyrene's but not for benzo[a]pyrene's induction of p53 phosphorylation and apoptosis. Toxicology. 2008; 244(2-3):257-270.

33. Cheng WH, Zheng X, Quimby FR, Roneker CA and Lei $\mathrm{XG}$. Low levels of glutathione peroxidase 1 activity in selenium-deficient mouse liver affect c-Jun N-terminal kinase activation and p53 phosphorylation on Ser-15 in pro-oxidant-induced aponecrosis. Biochem J. 2003; 370(Pt 3):927-934.

34. Sablina AA, Chumakov PM, Levine AJ and Kopnin BP. p53 activation in response to microtubule disruption is mediated by integrin-Erk signaling. Oncogene. 2001; 20(8):899-909.

35. She QB, Bode AM, Ma WY, Chen NY and Dong Z. Resveratrol-induced activation of p53 and apoptosis is mediated by extracellular-signal-regulated protein kinases and p38 kinase. Cancer Res. 2001; 61(4):1604-1610.

36. Rasul A, Ding C, Li X, Khan M, Yi F, Ali M and Ma T. Dracorhodin perchlorate inhibits PI3K/Akt and NF-kappaB activation, up-regulates the expression of p53, and enhances apoptosis. Apoptosis. 2012; 17(10):1104-1119.

37. Park S, Kim D, Dan HC, Chen H, Testa JR and Cheng JQ. Identification of an Akt interaction protein, PHF20/TZP, that transcriptionally regulates p53. J Biol Chem. 2012; 287:11151-11163.

38. Weng JR, Bai LY, Chiu CF, Wang YC and Tsai MH. The dietary phytochemical 3,3'-diindolylmethane induces G2/M arrest and apoptosis in oral squamous cell carcinoma by 
modulating Akt-NF-kappaB, MAPK, and p53 signaling. Chem Biol Interact. 2012; 195(3):224-230.

39. Dung TD, Day CH, Binh TV, Lin CH, Hsu HH, Su CC, Lin YM, Tsai FJ, Kuo WW, Chen LM and Huang CY. PP2A mediates diosmin p53 activation to block HA22T cell proliferation and tumor growth in xenografted nude mice through PI3K-Akt-MDM2 signaling suppression. Food Chem Toxicol. 2012; 50(5):1802-1810.

40. Manfe V, Biskup E, Rosbjerg A, Kamstrup M, Skov AG, Lerche CM, Lauenborg BT, Odum N and Gniadecki R. miR-122 regulates p53/Akt signalling and the chemotherapy-induced apoptosis in cutaneous T-cell lymphoma. PLoS One. 2012; 7(1):e29541.

41. Lee KB, Byun HJ, Park SH, Park CY, Lee SH and Rho SB. CYR61 controls p53 and NF-kappaB expression through $\mathrm{PI} 3 \mathrm{~K} / \mathrm{Akt} / \mathrm{mTOR}$ pathways in carboplatin-induced ovarian cancer cells. Cancer Lett. 2012; 315(1):86-95.

42. Ji H, Ding Z, Hawke D, Xing D, Jiang BH, Mills GB and Lu Z. AKT-dependent phosphorylation of Niban regulates nucleophosmin- and MDM2-mediated p53 stability and cell apoptosis. EMBO Rep. 2012; 13(6):554-560.

43. Del Sal G, Murphy M, Ruaro E, Lazarevic D, Levine AJ and Schneider C. Cyclin D1 and p21/waf1 are both involved in p53 growth suppression. Oncogene. 1996; 12(1):177185.

44. Boehme KA, Kulikov R and Blattner C. p53 stabilization in response to DNA damage requires Akt/PKB and DNA-PK. Proc Natl Acad Sci USA. 2008; 105(22):7785-7790.

45. Astle MV, Hannan KM, Ng PY, Lee RS, George AJ, Hsu AK, Haupt Y, Hannan RD and Pearson RB. AKT induces senescence in human cells via mTORC1 and p53 in the absence of DNA damage: implications for targeting mTOR during malignancy. Oncogene. 2012; 31(15):1949-1962.

46. Rao B, van Leeuwen IM, Higgins M, Campbel J, Thompson AM, Lane DP and Lain S. Evaluation of an Actinomycin D/ VX-680 aurora kinase inhibitor combination in p53-based cyclotherapy. Oncotarget. 2010; 1(7):639-650.

47. van Leeuwen IM, Rao B, Sachweh MC and Lain S. An evaluation of small-molecule p53 activators as chemoprotectants ameliorating adverse effects of anticancer drugs in normal cells. Cell Cycle. 2012; 11(9):1851-1861.

48. Huang MC, Chen FY, Chou MT and Su JG. Fluoranthene enhances p53 expression and decreases mutagenesis induced by benzo[a]pyrene. Toxicol Lett. 2012; 208(3):214224. 